\title{
TU/e EmonOWEN

\section{What's the Situation with Situated Visualization? A Survey and Perspectives on Situatedness}

\section{Citation for published version (APA):}

Bressa, N., Korsgaard, H., Tabard, A., Houben, S., \& Vermeulen, J. (2022). What's the Situation with Situated Visualization? A Survey and Perspectives on Situatedness. IEEE Transactions on Visualization and Computer Graphics, 28(1), 107-117. [9552238]. https://doi.org/10.1109/TVCG.2021.3114835

DOI:

10.1109/TVCG.2021.3114835

Document status and date:

Published: 01/01/2022

\section{Document Version:}

Accepted manuscript including changes made at the peer-review stage

\section{Please check the document version of this publication:}

- A submitted manuscript is the version of the article upon submission and before peer-review. There can be important differences between the submitted version and the official published version of record. People interested in the research are advised to contact the author for the final version of the publication, or visit the $\mathrm{DOI}$ to the publisher's website.

- The final author version and the galley proof are versions of the publication after peer review.

- The final published version features the final layout of the paper including the volume, issue and page numbers.

Link to publication

\section{General rights}

Copyright and moral rights for the publications made accessible in the public portal are retained by the authors and/or other copyright owners and it is a condition of accessing publications that users recognise and abide by the legal requirements associated with these rights.

- Users may download and print one copy of any publication from the public portal for the purpose of private study or research.

- You may not further distribute the material or use it for any profit-making activity or commercial gain

- You may freely distribute the URL identifying the publication in the public portal.

If the publication is distributed under the terms of Article 25fa of the Dutch Copyright Act, indicated by the "Taverne" license above, please follow below link for the End User Agreement:

www.tue.nl/taverne

Take down policy

If you believe that this document breaches copyright please contact us at:

openaccess@tue.nl

providing details and we will investigate your claim. 


\title{
What's the Situation with Situated Visualization? A Survey and Perspectives on Situatedness
}

\author{
Nathalie Bressa, Henrik Korsgaard, Aurélien Tabard, Steven Houben, Jo Vermeulen
}

\begin{abstract}
Situated visualization is an emerging concept within visualization, in which data is visualized in situ, where it is relevant to people. The concept has gained interest from multiple research communities, including visualization, human-computer interaction (HCl) and augmented reality. This has led to a range of explorations and applications of the concept, however, this early work has focused on the operational aspect of situatedness leading to inconsistent adoption of the concept and terminology. First, we contribute a literature survey in which we analyze 44 papers that explicitly use the term "situated visualization" to provide an overview of the research area, how it defines situated visualization, common application areas and technology used, as well as type of data and type of visualizations Our survey shows that research on situated visualization has focused on technology-centric approaches that foreground a spatial understanding of situatedness. Secondly, we contribute five perspectives on situatedness (space, time, place, activity, and community) that together expand on the prevalent notion of situatedness in the corpus. We draw from six case studies and prior theoretical developments in $\mathrm{HCl}$. Each perspective develops a generative way of looking at and working with situatedness in design and research. We outline future directions, including considering technology, material and aesthetics, leveraging the perspectives for design, and methods for stronger engagement with target audiences. We conclude with opportunities to consolidate situated visualization research.
\end{abstract}

Index Terms-Situated visualization, literature survey, situatedness.

\section{INTRODUCTION}

In the last decade, situated visualization [defined in $64,100,105]$ has emerged as a research area and a concept that continues work initiated in the area of Ubiquitous Computing (ubicomp) and Human-Computer Interaction (HCI) [37, 81, 88, 98]. The central idea is to bring data visualizations into their context of use, to places and people by connecting data with the physical environment or physical referents that the data refers to $[100,105]$. The area of situated visualization follows a strand of research agendas in visualization that are concerned with moving beyond traditional desktop applications [75] such as "Ubiquitous Analytics" [26], "Immersive Analytics" [3, 29], or "Situated Analytics" [27, 90]. The literature on situated visualization spreads across multiple communities and research areas including HCI, visualization, Augmented Reality (AR), ubicomp, and urban computing, as well as subtopics such as public visualization and data physicalization. This wide appropriation of situated visualization as a research concept has led to a disconnected terminology, implications, and visualization design, creating a highly fragmented and inconsistent research landscape. As a result, interpretations of what situatedness and situated visualization are, and how these concepts are understood in the current literature, remain unclear.

To investigate how the research community has adopted and used the concept of situated visualization, and to broaden the scope of situatedness, we present a two-fold literature review and case study analysis. In the first part of the paper, we contribute an analysis of the literature that explicitly uses the term "situated visualization". We discuss and reflect on how these papers define situated visualization, the methods

- Nathalie Bressa is with Aarhus University, Denmark. E-mail: nathalie.bressa@cc.au.dk.

- Henrik Korsgaard is with Aarhus University, Denmark. E-mail: korsgaard@cs.au.dk.

- Aurélien Tabard is with Université Claude Bernard Lyon 1, LIRIS, CNRS UMR5205, F-69621, France.E-mail : aurelien.tabard@univ-lyon1.fr.

- Steven Houben is with Eindhoven University of Technology, Netherlands. E-mail: s.houben@tue.nl.

- Jo Vermeulen is with Autodesk Research, Canada and Aarhus University, Denmark.E-mail: jo.vermeulen@autodesk.com.

Manuscript received xx xxx. 201x; accepted xx xxx. 201x. Date of Publication xx xxx. 201x; date of current version xx xxx. 201x. For information on obtaining reprints of this article, please send e-mail to: reprints@ieee.org. Digital Object Identifier: $x x . x x x x / T V C G .201 x . x x x x x x x$ and technology they use, the application domains of proposed situated visualizations, and the type of data and type of visualization. From an analysis of the corpus, we conclude that work within visualization and visual analytics using AR technology to place visualizations within the physical environment and using that environment as a direct visual reference is the most prevalent way of presenting situated visualizations in the corpus. A smaller strand of research focuses on community-oriented research within HCI by engaging with and displaying information within a community where data is collected. This illustrates the breadth of situated visualization research and the different ways of approaching and implicitly considering the role and importance of 'situatedness' and 'visualization' respectively. Upon closer analysis, we find that while situated visualization combines concepts and approaches from $\mathrm{HCI}$ and information visualization, current work has adopted and operationalized a mostly spatial understanding of situatedness.

In the second part of the paper, we present five perspectives on situatedness that include and expand on the dominant spatial understanding of situated visualization, building on concepts from ubicomp and HCI: space, time, place, activity and community. We illustrate these five perspectives with a case study analysis, introducing six case studies of situated visualizations that stem from the corpus and from selected examples to encourage the research community to think more systematically about broader concepts of situatedness. Through the case studies, we demonstrate how the perspectives can help to explicitly consider aspects of the situation in which the visualization is shown beyond spatiality alone and to recognize opportunities for a wider set of possible technologies beyond AR. We end with future directions, discussing how to leverage the five perspectives and consider methods for stronger engagement with target audiences, design considerations around technologies, and material and aesthetics, concluding with research opportunities to further strengthen the area of situated visualization.

\section{APPROACH}

The goal of this paper is to clarify the concept of situated visualization by using a two-fold approach. In a first stage, we examine the literature that currently self-identifies as situated visualization through a keyword-based literature survey. In the second stage, we look at the broader literature and analyze a selected set of case studies on situated visualization that, while they may not directly use the term, can be classified as concerning situated visualization and expand on the notions of situated visualization as covered in the corpus from the first stage. We use these case studies to exemplify five perspectives on situatedness 
that are based on concepts from ubicomp and HCI.

Given the variety and spread of the literature, we considered two strategies to analyze the literature on situated visualization: an openended interpretive approach and a keyword-based approach [25]. An open-ended interpretive approach examines research and examples based on the authors' knowledge of the research area and may include articles that could be analysed and interpreted as concerning situated visualization without necessarily being published with that focus. While this approach generates a broad and exploratory corpus, it also introduces subjective and less obvious inclusion and exclusion criteria and may introduce selective bias early in the process, as well as imposing a characterization that is not that particular publication's intended focus A keyword-based approach is inclusive towards papers published under the theme of situated visualization and provides a clear cutoff. However, in topics that are new and emerging or cut across multiple communities with different terminology, it can yield a relatively small initial corpus and it is subject to trends and competition in keywords. To combine the strengths of both approaches, we have applied a keyword-based approach in the first stage (the literature survey) and apply an openended interpretative approach in the second part (the case studies). We tested different strategies for our keyword-based approach, including the use of seed papers of common definitions of situated visualization (e.g. $[100,105])$. However, we found that there was a wide diversity of papers that cited those seed papers, including many papers that do not engage with the concept of situated visualization but rather focus on other aspects (such as AR applications). As a result, the papers citing those seed papers either did not fit our inclusion criteria or would already have been included, so this approach did not yield any additional valuable results.

For the second stage, we selected case studies based on our findings in the literature survey of the first stage. Our analysis of the current literature on situated visualization resulted in several themes, for example, in terms of applications, technologies, and definitions of situated visualization. We then curated and selected case studies that further broadened, expanded upon and provided contrasting perspectives on these themes to arrive at a broader categorization of situated visualization. The use of a curated set of case studies and notable examples to characterize and map out an emerging research area is a valuable approach that has been used before for casual information visualization [69], anthropographics [65], personal information visualization [45], and data physicalization [47]. The perspectives we develop are based on our analysis of the corpus as well as theoretical developments in HCI and ubicomp that we have selected to broaden the current understanding of situatedness for situated visualization.

\section{A Survey of Situated Visualization Research}

We survey a set of representative papers from a range of different research communities based on a keyword-based literature search to give an overview of current research in situated visualization.

\subsection{Corpus}

To build our corpus of papers, we collected a list of 44 core papers with the following inclusion criteria.

Keyword search: we performed a keyword search in the ACM Digital Library, IEEE Xplore, and Google Scholar for papers that contain the term "situated visualization". We focused our keyword search on one search term to get an overview of research that self-identifies as "situated visualization" and to find out how different communities currently use the term without imposing a categorization on papers While the resulting corpus did not include all possible publications that could potentially be classified as situated visualization, we considered this a reasonable trade-off in line with our goals. We also considered different regional spellings of the word "visualization".

Archival publications: we included full or short archival conference papers, journal and magazine articles, and book chapters. We excluded posters and workshop papers to focus on mature research contributions. Due to the broad list of communities in which situated visualization research is published, we did not restrict papers to particular venues. Therefore, our corpus includes a broad range of venues (including among others CHI, VIS, AVI, DIS, and ISMAR).

Relevance: We excluded papers that only showed up in the keyword search because they cite papers with "situated visualization' in the title, and do not otherwise mention situated visualization in the paper. We also excluded papers that only refer to situated visualization briefly in related work and do not further engage with the concept. We included all types of data representations that came up with the keyword search in the corpus, including both visualizations and physicalizations.

\subsection{Analysis}

We analyzed and coded the corpus in several iterations. First, we started by determining initial coding categories based on coding a subset of representative examples of the corpus. After this first iteration, all authors collectively discussed the coding schema and the corpus of papers. We identified additional dimensions, and merged and subdivided existing dimensions where relevant. Next, all authors engaged in focused coding on all the papers in our corpus, iteratively refining and revisiting the coding schema where necessary. After collectively coding all the papers in our corpus, we ended up with five dimensions that we use to categorize each of the papers (Table 1). Similar to Fonnet et al.'s [33] survey on immersive analytics, we included dimensions such as technology, data, and evaluation methods. The sub-categories of the dimensions were developed iteratively based on the corpus. The corpus was divided among all authors, in which the top-level categories were initially coded as free-text. Afterwards, each category was assigned to one author who then analyzed the categories holistically to develop the final set of sub-categories. Based on the coded papers in the corpus, we distilled a set of findings where we discuss how the term situated visualization is currently used in the literature, the technology and methods employed, the range of different domains in situated visualizations, and visualization and data types.

\subsection{Findings}

The corpus consists of a total 44 papers that we analyzed based on a set of five primary dimensions (see Table $1^{1}$ ). The dimensions include situated visualization definition, technology, type of data, method, and type of visualization. We further describe our findings of the coding to map out the space of current research on situated visualization. Regarding publication venues, the corpus covers 27 different venues. We can categorize these and their respective communities in the following broad groups: Visualization and Visual Analytics (e.g. IEEE VIS, IEEE TVCG, Information Visualization, PacificVis, AVI, Journal of Visual Languages \& Computing), HCI and design (CHI, DIS, Graphics Interface, Int. J. Human-Computer Studies), AR and 3D User Interfaces (3DUI, ISMAR, Handbook of Augmented Reality), Pervasive Displays, and Architecture. This illustrates that several academic communities engage in research on situated visualization, with the visualization and visual analytics, HCI and design, and the AR and 3DUI communities being the most prominent.

\subsubsection{Definitions of Situated Visualization}

The papers in the corpus can be split into papers that either define or expand the notion of situated visualization (column "Own definition" in Table 1) and papers that cite prior papers that defined situated visualization to frame their research. We categorized the papers based on which papers they cite and what definitions they use for situated visualization (see Table 1). While some papers cite multiple papers that contain definitions of situated visualization, we only considered the papers that were used to explicitly refer to situated visualization. For example, papers referring exclusively to the system contribution in SiteLens [100] are not categorized as using the definition.

The two most prevalent definitions of situated visualization cited in the corpus are White and Feiner's definition [100] (in some cases also White et al. [101]) and Willett et al.'s definition [105]. A total of 27/44

\footnotetext{
${ }^{1}$ Survey data: http://situatedvisualization.github.io/survey/
} 


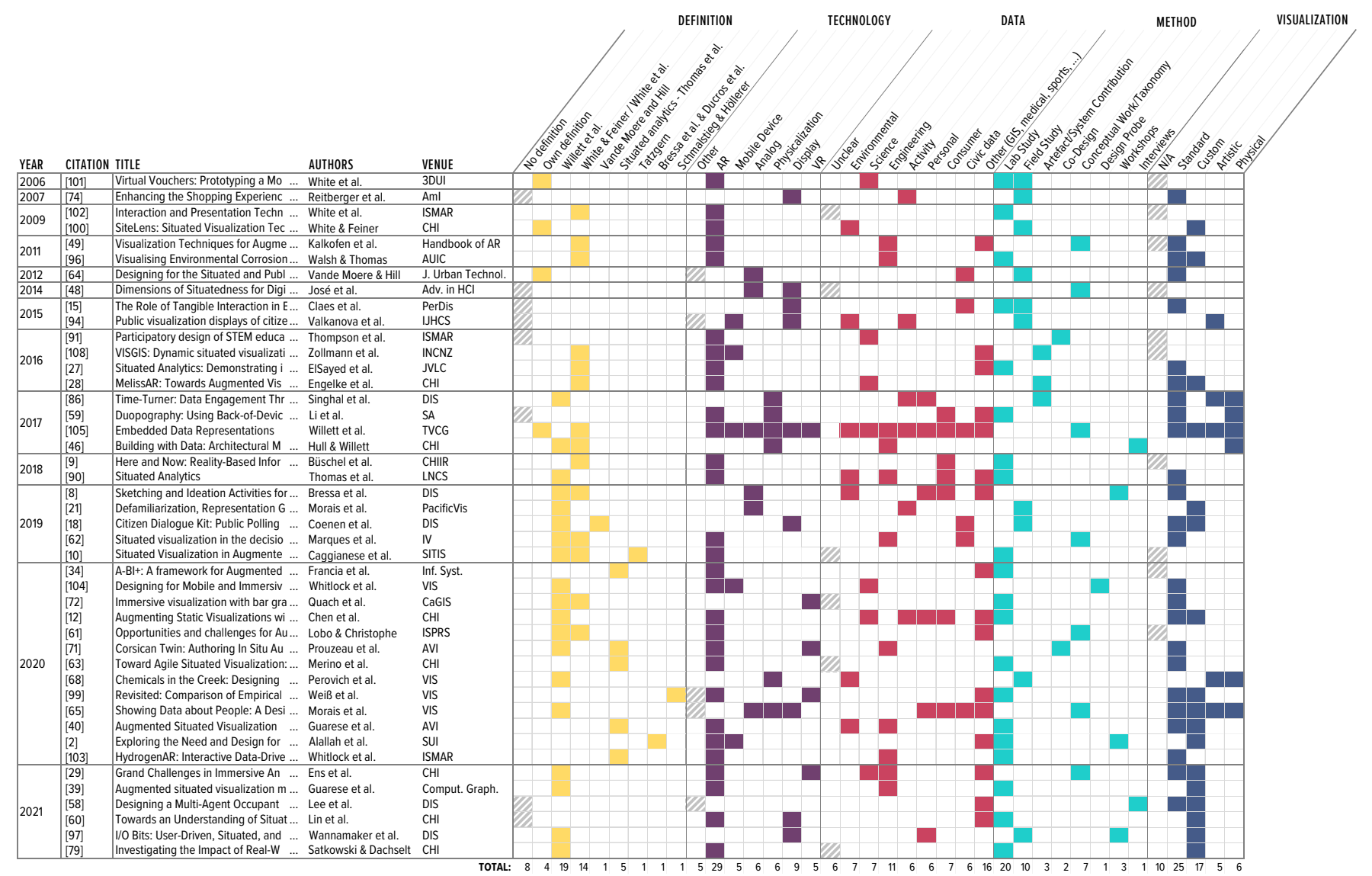

Table 1. Corpus of 44 papers coded by the categories: situated visualization definition; technology; data; method; and type of visualization.

papers cite either White and Feiner (8/44), Willett et al. (12/44) or both definitions (6/44). White and Feiner offer a broad definition of situated visualization as a visualization that "is related to and displayed in its environment" [100]. Willett et al. build on the work of White and Feiner by introducing the concept of a physical data referent, which the data is displayed in proximity to [105]. They make the distinction between situated and embedded visualizations and data representations. The key difference here is that embedded visualizations position presentations of data as close as possible to the physical referent, whereas situated visualizations, in Willet et al.'s definition, "place the entire visualization in a relevant location, but do not necessarily physically align individual data presentations or visual marks with their corresponding referents"'[p.464 105]. Hence, the distinction between situated and embedded, and the defining difference for both, are details regarding distance between referent and representation, and accuracy and fidelity in this relation.

In addition to these two prevailing notions of situated visualization, five papers in the corpus refer to situated visualization as part of situated analytics $[27,90]$. Situated analytics leverages AR together with visual analytics to support situated understanding and decision-making [27] and builds upon White and Feiner's work on situated visualization by offering a technology-centric view on situated visualization. Schmalstieg and Höllerer (1/44) [82] similarly describe situated visualization in the context of $\mathrm{AR}$ and refer to White and Feiner while pointing out that situated visualizations have a semantic meaning in the real world. Tatzgern [89] (1/44) provides a more open extension of White and Feiner, where "situated visualization is this connectedness of the information to the real world" [89, p.8]. Vande Moere and Hill (1/44) [64] offer a definition of situated visualization in the context of public and urban visualization. Just as previous definitions, situated means that the visualization is embedded in a real-world, physical environment [64, p.41]. However, where White and Feiner and Willett et al. focus on the physical features of the environment, Vande Moere and Hill focus on the broader meanings of the location along three dimensions defining situatedness as contextual, local, and social.

Apart from the papers that are cited as definitions of situated visualization, there are a number of papers in the corpus that further engage with the term situated visualization and the concept of situatedness including Büschel et al. [9] who outline the concept of reality-based information retrieval, Lobo and Christophe [61] who extend the concept of a physical referent with a geographical referent, and José et al. [48] who define six dimensions of situatedness for public displays, focusing on public signs and displays rather than situated visualization.

\subsubsection{Type of Technology}

We coded the corpus based on the type of technology used to show situated visualizations. Notably, several papers mention or include multiple technologies, for instance, papers with theoretical contributions like Willett et al.'s [105] paper on embedded data representations mention a range of technologies. As with the definitions of situated visualization, there is a similar dominance of AR work (29/44), with the remaining papers in the corpus looking at and developing more traditional display-based examples and analog and physical data representations.

The close relationship between AR research and situated visualization can be attributed to several possible reasons. First, the defining work by White et al. [100, 101, 102] has had some influence on subsequent work, not only in how the definition is used, but also in how SiteLens [100] has become a common example and use-case to refer to $[62,96]$. Second, within the AR and immersive analytics research communities, situated visualization is often seen as a use case for AR or a motor theme within AR $[49,85,108]$ and perhaps not as a research area in its own right. Hence, multiple works use situated visualization as part of making technical contributions to AR research [71] or immersive analytics [104]. Third, there is work on situated visualization in which AR satisfies specific requirements for the use case, such as the ability to superimpose information about structures within the built environment [96], underground utility infrastructure [80, 100], support for mobility $[59,101]$, search and access to task related in- 
formation $[9,10,40,104]$, or visualization of a phenomena close to an object, e.g. visualizing electromagnetic fields close to the object generating it [39].

Moving outside of AR-centric work, there is a broader catalogue of examples that include different technologies. A number of papers in the corpus (9/44) take an interest in exploring situated visualizations using more traditional display technology and another group (5/44) takes an interest in mobile devices. This includes several projects with a focus on displaying visualizations within semi-public and public spaces to engage people within proximity around locally collected data $[19,48$, $74,94]$. Another part of the corpus, focuses on showing data through an analog medium (6/44) or as data physicalizations (6/44). Two of the projects present designs where the visualization is part of a physical object: a custom clock [21] and coasters [86] that show visualizations of people's activity. In these examples, the visualization is designed to fit the physical object and vice versa. Hull and Willett [46] use wood to integrate data into architectural modelling and Perovich et al. [68] use lanterns to visualize pollution violations in a local creek. These projects in the corpus focus on situating visualizations with and through objects and with consideration towards the place and more ephemeral engagements with the visualization. In addition, the work takes on a technologically inclusive perspective where it is the place that is instrumented, rather than the people (e.g. not requiring the use of head-mounted displays to view the situated visualizations). Finally, an important point in several of these projects that do not rely on AR is to make data public which in turn suggests technologies that can be viewed by everyone within proximity so that the visualization can become part of a shared reflection or public debate $[19,64,68]$.

\subsubsection{Type of Data and Application Domains}

The type of data provides an overview of prominent types of data for situated visualization and common application domains.

One group of papers pertains to science and engineering applications. This includes engineering data (11/44) such as Building Information Modeling (BIM) and maintenance data [71, 103] or data on environmental corrosion [96]; environmental data (7/44) such as air pollution [100] or water permit violations [68]; and science data (7/44) such as honey bee behaviour [28], geological science data [104], or science education [91].

Another cluster of papers deals with situated visualization of data for the general public (19/44). This includes civic data such as public visualization of urban and locally-relevant data $[18,64,94]$ and public data installations [15]; personal data such as visualizations in domestic settings [86, 97]; and consumer data in domains such as shopping [74]. Related to this cluster of papers are a number of articles that focus on visualizing activity data, in the home [86] or in workplaces [21].

Finally, there are data types and application domains that only occur a few times, which we grouped in the other category (16/44). This includes GIS data [59, 108], medical data [49, 58, 90], education [12], sports $[2,60]$, business data such as logistics data in warehouses [8, 34] and instructions for DIY tasks [99].

Note that for several articles in the corpus, the type of data or target application domain was unclear (6/44). These articles often present multiple possible applications [48] or domain-agnostic techniques [10, $63,72,79,102]$. Overall, our analysis shows that situated visualization has been explored for a wide variety of data from different application domains and for different settings. However, the level of depth in engaging with a particular domain varies widely. Some papers in the corpus engage in depth with a particular target audience and application domain, through initial engagement with a domain, iterative prototyping and design, and a real-world deployment. However, this is rather rare, as most application domains are primarily used as illustrative examples of the visualization technique. As seen in the method column (Table 1), the majority of papers in the corpus rely on lab studies (20/44), with a number of field studies (10/44) and only a handful of papers that involve co-design (2/44), design probes (1/44) or workshops (3/44).

\subsubsection{Type of Visualization}

In terms of the type of visualization used, we found that a large number of papers in the corpus provide little to no reflection on their visualization design rationale. For about a quarter of the papers (column N/A: 12/44), it would be a stretch to categorize them as a deliberately designed data visualization. Instead, these papers often only visualize single data points in-place. An example of this is Zollmann et al. [108], which only shows text labels for buildings. As a notable exception to this, the work of Morais et al. [21], discusses in detail how the data is represented and how the (custom) visualization was designed.

The most common visualizations are what we have referred to in Table 1 as Standard visualizations-those that can be easily generated using common tools such as spreadsheets. The majority of the corpus (25/44) relies on such standard visualizations such as line charts, bar charts, or heat maps. The second largest group (17/44) in the corpus use Custom visualizations-handcrafted or custom designed visualizations specific to the application domain, the context, target audience or the data that is being visualized. A small number of papers rely on what we categorized as Artistic visualizations (5/44)-visualizations that show data in an aesthetically pleasing way, where the data visualization remains in the periphery when it is not the primary focus of attention for exploration and analysis. This category is related to ambient displays, and is inspired by Redström et al.'s informative art [73] and Stasko's information art [87]. Examples in the corpus are Chemicals in the Creek [68], Time-Turner [86] and Valkanova et al.'s Gurgle water fountain display [94]. For example, in Time-Turner, data is visualized on physical drink coasters augmented with LEDs. While the coasters can be used to explore the data, the visualization was designed to invite curiosity and be visually unobtrusive. Finally, some papers in our corpus are surveys or cover a wider design space and discuss several visualizations and thus provide multiple visualization types [65, 105].

In summary, the corpus shows limited engagement with how the data is visualized. Few papers deliberately discuss how the visualization was designed and how it represents the data. The majority of papers in the corpus either use standard visualizations or only visualize single data points (35/44). This could indicate that the area of situated visualization relies on prior innovations in visualization by leveraging well-known and standard visualizations, and rather focuses on how the data visualization is situated instead of on its representation. On the other hand, this may also point to a lack of concern for how the visualization should be designed to be presented in a situated manner. Indeed, some of the counterexamples (e.g. [21, 68, 86]) employ a carefully designed visualization to ensure appropriateness for the intended target audience and context of use.

\subsection{Summary of Findings}

Next, we summarize our key findings from the literature survey.

Limited engagement with definitions. We observed that few papers in the corpus engage deeply with the existing definitions of situated visualization (Sect. 3.3.1). Most papers merely cite one of the prevailing definitions (White \& Feiner [100] or Willett et al. [105]), but do not discuss in detail how they understand situatedness and situated visualization.

Limited engagement with how data is represented. We found that there is limited engagement with how data is represented and visualized in the corpus. Additionally, the majority of the corpus use standard visualizations, visualization as single data points or only information, or use visualizations as exemplars to showcase technical contributions. This may be also partly due to the view of almost any AR application being synonymous to situated visualization (e.g. ElSayed [27] and Schmalstieg and Höllerer [82]).

Limited engagement with target audience. There is limited engagement with and consideration of the target audience for the visualizations that are described in the corpus. This ties back to the prominence of lab studies as the most common way to study situated visualization in the corpus. Only a quarter of the papers in our corpus rely on field 
studies, and an even smaller set uses multiple methods, such as initial engagements through workshops or deployments and field studies.

AR as the dominant technology. From a technology perspective, the vast majority of papers in our corpus relies on AR, with the rest of the corpus utilizing other technologies such as larger displays, mobile devices, or physicalizations.

A spatial understanding of situatedness. There is a close relationship between situated visualization and AR which has influenced the field and emphasizes technical contributions. This leads to a prevailing spatial notion of situatedness in the corpus which emphasizes spatial properties and relationships between visualizations, objects, and locations.

\section{EMERGING PERSPECTIVES OF SITUATEDNESS}

Our survey confirms that papers on situated visualization conceptualize situatedness primarily as a set of properties related to space and proximity between the visualization and objects or features of the environment.

Starting with applying and operationalizing known features - in this case location and spatial organization - before exploring additional perspectives, is a common strategy in research. For instance, early work within context-aware and mobile computing started by investigating location tracking to provide novel applications and services. However, the community quickly realized that "there is more to context than location" [83]. Zimmermann et al. [107] make similar observations and explore five operational features of context. While these focus on categories of contextual information, their work illustrate the need for expanding early conceptions of context, and here situatedness, into more useful analytical and operational categories.

When exploring 'situatedness' beyond location and spatial relations, Dourish [22] made an important point: understanding context (and similarly situatedness) as a matter of spatial organization and cataloguing objects and relations reduces context and situation to a representational problem where information and (material) stability take precedence over the relational and dynamic aspects. Instead of being a product of metrics and spatial features, context arises from activity, in the sense that what meaningfully counts as context is what matters to the activity. This observation applies to understanding situatedness as well.

Suchman [88] made a related argument earlier when discussing the ability to model humans plans and reasoning: people can plan their activities but action is contingent on the conditions and environment in which it unfolds. Instead of conceptualizing use and action as carrying out intentional plans and following precise scripts, people act based on establishing a coherent and mutual understanding of action and meaning in unique material and social circumstances (situations). Suchman emphasizes language and social interaction as a primary mechanism for organizing activity. From this perspective, what 'is' the situation is a continuous achievement of social interaction. This is referred to as the practical objectivity of situations: when engaged in activities and social interactions we pay attention to what is important to the activity and interaction above less important aspects and things. Hence, what makes the situation mutually intelligible to the people involved in the activity is a product of how people make sense of activities and action through social interactions and language.

Based on these discussions on examinations of the additional factors and aspects of the context, and social and material circumstances that influence how visualizations can be considered situated, we introduce five perspectives of situatedness: space, time, place, activity, and community. We selected these perspectives based on broader theoretical discussions on situatedness in HCI, CSCW, and ubicomp and from emerging themes from the corpus analysis. The space perspective is derived from the largely spatial understanding of situatedness present in the corpus and Willett et. al.'s concept of physical referents. Place and activity are based on theoretical discussions on place in HCI (influenced by Dourish [23]) and activity theory [5]. The time and community perspectives are to a certain degree underdeveloped and implicit in the corpus (see Willett et al. [105] temporal indirection for time and work on public visualization for community $[19,68]$ ), which we develop further in relation to situatedness.
By introducing six cases (C1-C6), we illustrate how these perspectivescan be used. We will discuss and elaborate on the individual perspectives with the cases as examples.

\subsection{Exemplars of Situated Visualizations: Six Cases}

We analyse six cases $(\mathrm{C} 1-\mathrm{C} 6)$ from the literature we find to be good exemplars of situated visualizations and physicalizations to illustrate the different perspectives on situatedness we have identified (Figure 1). While these cases may not all use the term "situated visualization" explicitly, we introduce them to expand on the key concepts developed in the works of our initial corpus.

C1 Corsican Twin [71] is a VR authoring tool for designing situated and embedded AR visualizations remotely for building management. Based on the concept of digital twins that are digital replicas of realworld objects and spaces, the tool enables authoring of AR visualizations for large or difficult to access environments in a remote setting. People can create visualizations in VR in a 3D model of the interior of the target environment and afterwards view the created visualization in AR in the physical space through a head-mounted display.

C2 Situated Glyphs are small context-aware displays that show activity-specific information [51,95] to support work activities of healthcare professionals. The project explored the use of small wearable, mobile, or fixed displays that show relevant information to support interleaved activities involving multiple individuals and different types of equipment in the context of healthcare work.

C3 Cairn is "a tangible apparatus that enables situated data collection, visualization and analysis" of FabLab activities [36]. Its creators present it as an alternative to questionnaires and data collection techniques, while also materializing FabLab activities and fostering reflection on them. Cairn's physicalization consists in tokens visitors stack on a tabletop to record and display the type of activity they conducted in the FabLab, what resources it involved, what they learned and taught, and how much time they spent in the lab. The project has been implemented in a FabLab in Paris and has been adapted in the NYU Makerspace [56].

C4 Chemicals in the Creek [68] is a community-based physicalization in the form of an interactive installation. The project consisted of deploying connected lanterns representing water quality violations by industrial facilities during a public event. The installation itself was the result of two years of collaboration between researchers, citizens and activist networks, assembling data, deciding how and when to display it, and debriefing the event with the public afterwards.

C5 Activity Clock [21] is a wall clock that integrates data on the number of people typically present in a cafeteria of a research laboratory over a day. The visualization is made out of paper inside a plastic wall clock and has been deployed over the course of the week.

C6 Public Polling Displays [14] is a project about public visualizations on small distributed public polling displays that present a civic issue through data driven narratives, which has evolved into an interactive citizen participation survey display device [17]. In collaboration with a neighborhood committee in Antwerp, the researchers distributed multiple small e-ink displays on house facades of residents that presented perspectives on local issues consisting of a visualization of air pollution levels based on PM25 sensor data, a public polling question and results from the hosting resident, supporting infographics or textual annotations, and a personal statement of the hosting resident.

\subsection{Perspectives}

To expand the concept of situatedness, we introduce five key perspectives (space, time, place, activity, and community). While these five perspectives are complementary and could be considered for each of the case studies, we highlight selected case studies that are more prominent and relevant for particular perspectives than others. 

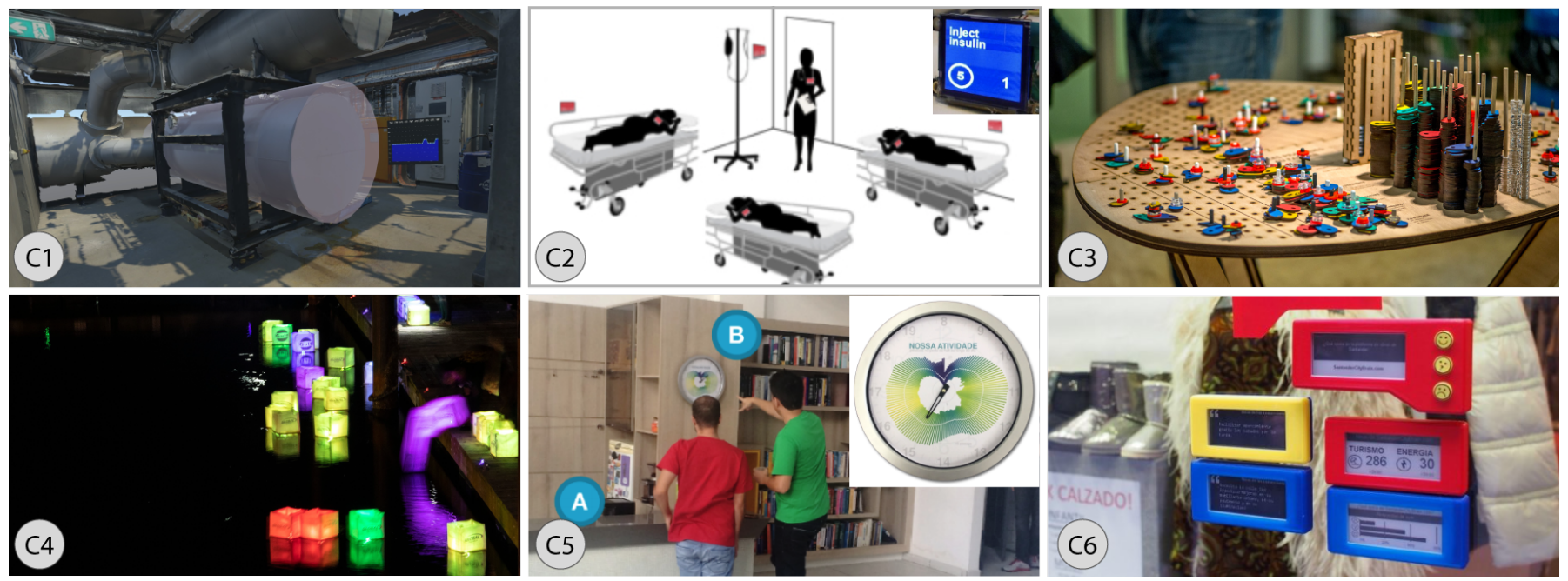

Fig. 1. Case studies: C1 Corsican Twin [71], @ 2021 A. Prouzeau; C2 Situated Glyphs [51, 95], ( 2012 IEEE; C3 Cairn [36], (@ 2021 P. Gourlet; C4 Chemicals in the Creek [68], () 2020 W. Campbell/IEEE; C5 Activity Clock [21], () 2020 IEEE; C6 Public Polling Displays [14, 17], ( 2021 J. Coenen.

\subsubsection{Space}

The space perspective is implicit to most works reviewed in Section 3, and focuses on the spatial organization and relationship between the physical environment and situated visualizations. Framing situatedness as a problem of spatial representation implies that the unit of analysis is the placement of visualizations with regards to spatial properties and features in a three-dimensional environment (e. g., location, proximity, distance, and physical structure). This spatial perspective resembles many similar attempts to sense and represent context in context-aware computing and recently in proxemic interaction [38].

The space perspective is explicitly represented in the key definitions by White and Feiner [100] and Willett et al.[105], in particular in Willett et al.'s concept of physical referents. They approach situatedness as a relationship between physical referents and physical representations of data. They position this relationship within an extension of the data visualization pipeline that includes both a physical and logical world where physical referents are a "physical object or physical space to which the data refers"[p.462 105]. The emphasis, thus, lies on the proximity between data representations and physical referents which is used to distinguish between situated and embedded visualizations and to determine the level of "spatial indirection".

While space, by definition, plays a role in all the cases, there are cases where it is a particularly useful perspective to consider that can be helpful to examine other aspects of situatedness. C1 (Corsican Twin) is a good example in which spatial situatedness is key in understanding the connection between the physical space, the visualization and the activity it is intended to support. In C1, visualizations about the equipment and the site are situated by pairing equipment with data streams from associated sensors. The visualizations, as overlays, create a direct relationship between data (such as volume in a tank, see Figure 1, C1) and physical referents (the machinery). Here, it is crucial that the spatial relationship is mapped as accurately as possible. However, as we further discuss in the activity perspective (4.2.4), closeness is not always desirable from a design point of view. For instance, when there is no direct spatial relationship to physical referents, or when the visualization blends different data from different referents, direct spatial closeness might not be optimal or even appropriate.

The Situated Glyphs project $\mathbf{C 2}$ distinguishes between three types of spatial placement of glyphs in the hospital: entity-centric, activitycentric and space-centric. Inspired by Pederson's situative space model [67], these three variations consider space beyond physical distance between the visualization and the entity of interest, recognizing that people's activities and context of use are important to consider.

Going beyond purely spatial relationships between data and physical referents that focus on displaying data where it is produced, Willett et al. [105] touch upon the concept of a semantic relationship between data and physical referents which introduces the importance of tasks and observational goals of the viewers and the meaning people put into situated visualizations. In the following sections, we expand on the spatial perspective on situatedness by introducing the additional perspectives of time, place, activity, and community to better consider how people make sense and interact with their environment.

\subsubsection{Time}

Taking a temporal perspective puts the emphasis on the relationship between when data is recorded and when it is presented. The projects reviewed in our survey tackle time in various ways, ranging from displaying only live data, to historical or cumulative visualizations. Willett et al. [105] note that, as for space, one can consider temporal indirection, defined as the distance "between the moment in time a physical presentation is shown and the original time it refers to". In Newtonian or naïve physics, this corresponds to the notion of a linear and directional flow of time, leading to a global ordering, and to observers sharing the same time reference [78]. Adopting a linear time-flow perspective means that to be situated, visualizations should minimize temporal indirection [105], i.e. display data as it is captured. This is a particularly strong design constraint, which is relaxed in most situated visualizations. Since one cannot physically go back in time, traces or cumulative data is often displayed instead.

Yet, temporal data can be structured and thought of in a variety of alternative non linear ways [1]. In C5 (Activity Clock), the situated visualization adopts a circular 12-hour clock view to represent the average number of people in a cafeteria at a given time of the day [21]. With data spanning three years aggregated in a static clock-like visualization, is it the daily cyclical rhythm of the cafeteria that is conveyed rather than a linear evolution. By setting up the display in the cafeteria, the authors situate the visualization in space, yet the interviews of observers also reveal that they interpreted the visualization reflecting on their temporal knowledge of local routines, of their individual habits, and of the social temporality of the cafeteria.

The question of temporal relevance, changes and synchronicity, has been important to social sciences scholarship. As sociology was defining itself as a discipline, in the early $20^{\text {th }}$ century, "social time" [41] became a central object of inquiry. This involved considering time as neither fully objective, nor subjective but rather socially constructed This enables to account for multiple temporalities that are constitutive of lived experiences. Social time can still be found in metrics, for instance, in agreed upon time zones, or shared $24 \mathrm{~h}$ time-frames, rather than reliance on solar time. Taking into account social time leads to another perspective on temporal situatedness. Temporal relevance (rather than proximity) relates to activities, shared cultural references and conventions, habits, needs for coordination. Temporal situatedness 
is constructed through the interactions between individuals, groups and their objects of interest. In $\mathbf{C 5}$, cafeteria breaks become temporal references, in between events and periods, they are activities associated with a place and with people, but also something of broader social and temporal meaning: a break in the workday and a time with associated experiences and cultural conventions.

In $\mathbf{C 3}$ (Cairn), the physicalization strategy consists of stacking tokens to represent events happening in a FabLab, with the most recent being on top [36]. The time of data capture and the time of visualization are blended, as the visualization is created by manually capturing events. More importantly, the physicalization creates a shared temporality inside the FabLab, as the manual assembly can also provoke encounters or discussions about recent events or distant ones, that resurface and become "present" through stories told around the table.

This aspect of shared experience is also important in C4 (Chemicals in the Creek). While the visualization is ephemeral, lasting only one evening, it exists within multiple time frames: the public event and the lifelong experience of pollution of local inhabitants. With the physicalization being created for this specific event, temporal situatedness could be considered high although the data captured is several months old. Moreover, as environmental pollution unfolds over very long time scales, the notion of temporal indirection [105] may not be the most relevant to assess the situatedness. The pollution sensed months ago may still be present in the environment years in the future.

To sum up, a linear or cyclical perspective on time, in which time is considered an objective non-reversible arrow, "flowing equably without regard to anything external" [78] can be useful. Yet in many cases, temporal situatedness has more to do with the way data connects to the temporal experience of the viewers.

\subsubsection{Place}

Taking a place perspective means paying attention to a location while breaking away from a purely spatial perspective and representational approach. A place is shaped by its history, its local identity and the meaning it has for its inhabitants [93]. Hence, visualizations become situated if they fit within and represent not only relevant data, but the unique aspects of a particular place, such as data collected by the inhabitants or local cultural heritage. Place as a perspective becomes particularly relevant in relation to the urban- and place-based visualizations that are contained in the corpus.

Several papers discuss aspects of situatedness in relation to and with reference to places. For instance, Vande Moere \& Hill [64], Valkonava et al. [94], and José et al. [48] all discuss aspects of place, local identity and culture in relation to situated visualizations. The meaning of place is usually contrasted with the meaning of space. Whereas space is the spatial structure of the world, a space becomes a place by gaining meaning through living in it, the people occupying the space, and its historical context. Harrison and Dourish describe the difference as "we are located in space, but we act in place" [42, p.69].

Examinations of place are frequent in HCI that build upon various traditions but share a common focus on how we experience place [13], place as a unit of analysis [32] and the challenges in designing for places [53]. All of these works introduce place as more than a simple backdrop or 'context' for activity, but as a meaningful relation people have to a locale through its socially and culturally embedded meaning.

In C6 (Public Polling Displays), the concept of place plays a role in how the displays are situated and how data is collected. To engage with the public polling displays, to vote on the presented local issues, and to understand displayed data, only makes sense if the person viewing the visualizations has a relation to the place in which they are deployed. People who have a relationship to the place are residents, neighbors, visitors, and daily commuters to that particular neighborhood who interpret the visualizations in hyper-contextual ways. These polls and displays have a dual role: they collect and display local information about the place, but they also define what is of local relevance, i. e. what is a place. It is through this interaction that the place takes its shape and the visualization becomes situated.

There are similar principles at play in $\mathbf{C 4}$ (Chemicals in the Creek). Here, the visualized data is important to the place where the installation is presented. While the data is not directly related to the dock where the installation and event took place, the place of the event created an opportunity to make people aware of how water permit violations influence the creek, the people, businesses, and homes. The project goal was increasing community ownership over the waterfront and creating a place for communication within the community. It is through the event, in a given location, with people sharing a matter of concern by creating an ephemeral place, that the physicalization becomes situated.

With C5 (Activity Clock), people's collective presence in the university cafeteria and general activity patterns in terms of coming and going are visualized on a wall clock. This visualization is designed for collective reflection and to support social engagement, which fits well with the character of the place in which it was deployed - which people frequent to get a drink and often socialize in. People's interest in the data is tightly coupled with the attachment to the place of deployment, situating the visualization by creating meaning to the locale.

Overall, the place perspective emphasizes situating data in within places that are relevant to people through their embedded socio-cultural meaning which provides opportunities to create meaningful relations between data and places.

\subsubsection{Activity}

From an activity perspective, situatedness implies that visualizations are not used in isolation but are embedded and connected to a wider set of human activities of target audiences. As visualizations move into everyday environments, designers need to consider why people conduct certain activities, how visualizations can meaningfully mediate these activities, and how they relate and connect to broader activities conducted across spaces, over longer time spans, or via collaborations.

These considerations of socio-cultural aspects of design have been developed in depth in HCI research through Activity Theory [50]. Activity Theory describes "activity" as a complex relationship between a person and their goals that is mediated by socio-cultural and historical context and tools - such as an interactive device. Because of this mediating function of technology, Bødker [5] notes that people do not act with but rather through computer interfaces. Interactions with computers are done to support or mediate real-life activities that involve shared practices with multiple people and different tools. The importance of people's activities in the situation at hand means that designing and evaluating situated visualizations requires a deep understanding of people's activities in a specific context as the relevant activities determine the appropriateness of particular designs, representations, placements, or technologies that are considered for the situated visualizations.

As an example, the Corsican Twin project C1 [71] takes into account common work activities of Building Management System (BMS) engineers and maintenance technicians when designing the situated visualizations. Examples of these activities include analyzing the current state of the system or the temporal evolution of specific variables. An initial study showed several benefits of the situated visualizations such as the usefulness of only showing localized information and showing documentation in-situ. However, later expert feedback highlighted how the activities of workers impacted spatial aspects of the situated visualization. For instance, some participants wanted to place visualizations near the sensors rather than near the equipment as that is where technicians would focus their attention first. Data about distant systems was also deemed useful to bring into the site for comparison and context. Several participants tended not to place visualizations on objects, but rather placed visualizations in $2 \mathrm{D}$ on nearby walls and surfaces to make the data more manageable. This shows how the work activities of people in different roles within the space guided the ways in which it was useful for them to situate data and visualizations which, in turn, influenced the placement of visualizations.

Similarly, Situated Glyphs (C2) [51, 95] are visualizations that are designed for a work context, in this case to assist nurses in a hospital setting. While the entity-centric, activity-centric and space-centric placements were informed by initial fieldwork in the ward, when conducting studies in the hospital with nurses and the prototypes, a number of additional issues emerged. The nurses were very positive about being shown overviews and information relevant to the activity at hand, and 
appreciated automatic documentation of performed activities. However, it was clear that the specific constraints of the workplace and the activities performed in it influenced many aspects of the design, in particular display placement. Entity-centric glyphs were not deemed to be as useful due to concerns about safety and practicality of wearable displays or displays that were fixed to objects or individuals. While hands-free operation was convenient, there were many issues with placement of displays around equipment that would often be moved around. The most promising and flexible solution seemed to be a hybrid mobile system that could be carried around and placed in an appropriate location while performing activities, such as a display that could be attached to a wall. Moreover, it was crucial to remain in control and be able to cope with unexpected situations and frequent interruptions. This case shows that a variety of aspects surrounding individual and collaborative activities as well as shared practices in the workplace determine the appropriateness of certain technology, placements, and visualizations.

Overall, these two cases illustrate that there are several aspects of situatedness related to people's activities - such as shared work practices, social and safety protocols, unexpected situations and interruptions that go beyond spatial aspects and can have an important influence on the suitability of different spatial layouts. These case studies highlight that a deep understanding of people's activities is important so that the technology and visualizations can be properly appropriated and integrated with people's existing activities.

\subsubsection{Community}

Taking a community perspective puts emphasis on the community of people who are the audience for and/or co-creators of visualizations centered around local issues and shared concerns [e.g. 20]. This perspective supplements place and activity by empirically foregrounding the community and local issues. Existing work on situated visualization that considers a specific group or community as the audience of situated visualizations focuses on "anchoring" the situated visualization within a specific community. Focusing on how a particular community uses and appropriates technologies in their activities is a common topic in HCI research $[6,11]$, including work that explores engaging with (urban) communities and local issues[20] as well as research within public visualization $[64,84,94]$. The community perspective is closely related to the place and activity perspectives as a place gets its meaning over time with and through a community and the activities that are happening within that community. The perspective of community is concerned with initiatives that focus on supporting a community in its activities, and using technology to support coordination around a shared concern. In projects that focus on supporting communities through situated data visualization such as $\mathbf{C 4}$ (Chemicals in the Creek) or $\mathbf{C 6}$ (Public Polling Displays), community members are involved throughout the design process, starting with early engagements to understand matters of concern, and then transitioning to activities that actively involve community members in the design of the technology, typically using participatory design or co-design methods. In the context of public visualization, Schoffelen et al. [84] discuss how visualizations can reveal the backstories behind facts from different perspectives, which when carefully designed to be readable and transparent - can allow for engaged discussions and reflections within a community.

In C6 (Public Polling Displays) [14], the situated visualizations were designed to address shared concerns around local issues that the community members found important, and were placed on house facades. In the study of the system with a neighbourhood committee for a street in the city of Antwerp, the shared concern was air pollution, with the neighbourhood committee aiming to get the street blocked for non-local traffic. Community members who hosted displays with visualizations on their facades could add their own backstories to the visualizations to support community-driven dialogues. The spatial location of the visualizations had an impact, but the social aspects of that location were just as important. For example, the perception of and trust in the visualized data was affected by the social relationship between the passerby and the visualization-hosting community member, either in a positive or negative way, depending on the hosting member's social standing with the passerby and in the community at large [14].
For $\mathbf{C 4}$ (Chemicals in the Creek) [68], the shared concern was raising awareness of spills and pollution in the Chelsea creek. The community that the researchers engaged with was GreenRoots: an environmental justice organization in Chelsea, Massachusetts. The researchers created a one-time performance to foster trust and connection among community members on the topic of water quality violations. The performance acted as a ceremonial event for witnessing data that supported shared reflection and recollection about the spills. Here, the situated visualization and the community build on each other: the visualization is situated partly because it relates to a shared community concern, and the visualization fosters community development.

In C3 (Cairn) [36], the physicalization targets the community around a FabLab in Paris and their shared concern is reflection on and documentation of the activities that happen in the FabLab over time. Gourlet et al. [36] note that Cairn enables "new collective reflections" and allows community members to discuss what they "are as a community in front of a landscape that describes our practices [i.e. Cairn] and with an experienced, thus, negotiable code.". Thus, Cairn acts as a facilitator around a shared interest of the members of the FabLab community involving the community members in the data collection process.

Overall, the community perspective emphasizes situating data in relation to communities of people to support engagement with local issues and concerns, often by involving the community in a participatory process.

\section{Discussion}

The perspectives we presented in the previous section are complementary ways of approaching situatedness. Space - as the most prominent perspective in the corpus of research we reviewed - is a useful starting point when designing situated visualizations as it provides the foundations for placing visualizations in the world. However, considering other perspectives on situatedness is key in addressing common challenges (see e.g. [29]) and enable designers to take into account broader considerations into their design, and improve the experience of situatedness for observers or users of the visualizations. Reflecting on our survey and the perspectives, we discuss technology, material and aesthetics, leveraging the perspectives for design as well as methods for stronger engagement with target audiences.

\subsection{Technological Considerations}

Norman controversially stated: "technology first, needs last" [66], arguing that technological innovation often leads to new conceptual inventions and the development of potential use cases. Our survey suggests that we may be witnessing a similar movement in situated visualization. A new wave of commercially available AR technology is sparking interest in the development of situated visualizations, which may lead to novel use cases. It is reminiscent of how the advent of mobile computing led to the emergence of ubiquitous computing.

While novel technology can enable novel use cases, it should, however, also not foreclose the use of alternative, sometimes more appropriate technologies. Although AR may be well suited to personal and private situated visualizations, collaborative AR technology is still in its infancy. Public displays may be better suited for collaborative, shared or public situated systems as such systems already support spontaneous interactions, do not require personal equipment, and can be used collaboratively. Static posters or data physicalizations do not need power, and can be made sturdy enough to be left alone for long time periods. E-ink displays have low power requirements and can be integrated inconspicuously into a variety of different environments $[8,14]$, while chalk's transience facilitates lightweight updates and local adoption [4, 52].

Work on situated visualization would benefit from engaging in deeper discussions on technological choices related to the case or target domain, without defaulting to one technology or another. Technology alone, whether it is AR or chalk on the street, does not make a visualization situated, solely on the basis that it displays data in proximity to its source. To situate visualizations into an environment, technological choices should consider the target audience and the context of use.

Moreover, these technological choices are conditioned by the underlying infrastructure that is available. To work smoothly, AR requires 
robust computer vision techniques and indoor location tracking in order to determine its location and recognize surrounding objects. As demonstrated in C1 (Corsican Twin), having access to richer building and object models enables much finer augmentations and situatedness. Likewise, public displays often require a wireless and electric infrastructure to be in place in order to be installed without problems. In other projects such as Mill Road [52], constraints on not touching the city infrastructure led to picking chalk rather than a more durable medium. Situated technologies, whether AR, public displays, or lower-tech, cannot be thought of without also considering how they will interface with the environment and the technical infrastructure they should fit in.

\subsection{Material and Aesthetic Considerations}

In addition to considering which technology to use, when fitting a visualization within a place, material properties also have to be considered. In C3 (Cairn), for instance, the artefact itself is fabricated in the FabLab so its material properties match with the surrounding space. In the area of public visualization, Street Infographics [16] integrates demographic information about a street into in the form of an addition to a street sign which uses the existing urban infrastructure to present information. Similarly, in projects like Mill Road [52] and Tidy Street [4], visualizations are drawn with chalk on the pavement and street, which integrates the visualizations with the urban environment. Fitting a visualization within the environment is not only a matter of the choice of technology or medium, but also of the visual representation itself. Rodgers and Bartram's [76] energy feedback visualizations show the value of an artistic and aesthetically pleasing representation of data that is designed to fit within a home environment to match with people's personal preferences. Similarly, C5 (Activity Clock) highlights the importance of an aesthetic fit for visualization designs within the space the data is displayed in. This is line with work on ambient displays [106], aesthetic requirements for information visualization [57] and projects on public information displays [94] that all show that material and aesthetic aspects are important considerations when designing visualizations that are to be situated and fitted within a specific environment. While there is a balance between choosing a technology and visual representation that offers the functionality needed and a good material fit within the environment, considering materiality is an important lens for choosing how to display and visually represent situated visualization.

\subsection{Leveraging the Perspectives for Design}

These perspectives do not suggest to simply examine new types of referents [105] for designing situated visualizations, such as for the community perspective, replacing physical objects as referents with community elements (actors, places, matters of concern). Our goal is to open up the discussion within the different research communities that use situated visualization to explicitly consider and more deeply engage with broader characteristics of situatedness beyond spatial aspects, as discussed in this paper. This contributes to expanding and clarifying the scope, definition and applicability of situatedness for visualizations.

Rather, we argue that the different perspectives of situatedness expand research stances on situated visualization and are beneficial to develop situated visualizations that are driven by their context of use. We position these perspectives as vantage points that supplement each other from a theoretical, methodological, and design angle. They play a role as starting points in design - who to talk to, where to go, what to consider - and act as deliberate generative strategies throughout the process. They invite participants and researchers to ask - what would be important to consider in terms of situating visualizations if space, time, community, activity or place is the primary unit of focus? Each perspective foregrounds different aspects of situatedness and focusing on one should be a deliberate choice in the design process. To account for the different perspectives when designing for situated visualization, new approaches and research in design methods are necessary [7].

To broaden the view on situatedness beyond spatial aspects alone, it is important to expand on methodological approaches. While lab studies are used to gain an understanding of how people interact with and perceive situated visualizations, in order to design and account for situatedness in all its richness (across the perspectives), it is essential to engage with the context and the audience the visualization is intended for. This often starts early on in the research process, by engaging in fieldwork to understand the target audience and the environment in which the visualization would be deployed, as we observe in several of our case studies (e.g. C4 [68] and C6 [14]). The realism of lab studies can also be improved by conducting them in an environment that closely resembles the eventual deployed environment, as with the on-campus research space with sensor data in Corsican Twin (C1) or studies in the hospital ward in Situated Glyphs (C2).

An ecologically valid methodology that situated visualization researchers could consider is "research in the wild" [77], i. e. creating and evaluating new technologies in-situ, observing how people react to the new technology and how they change and integrate the technology into their lives. Beyond evaluating a visualization design, many design constraints will also only become apparent by taking into account the environment in which the visualization will be deployed. This requires the use of and further research into developing in-situ (and participatory) design tools and methods, which some researchers have started exploring [8, 14, 24, 44, 95]. For example, Bressa et al. [8] explored the use of several situated design and visualization sketching exercises, including the use of magnetic whiteboard sheets and tiles mimicking the form factor of small displays. Ducros et al. [24] similarly explored situated design exercises and tools, where being in-situ provided tacit knowledge about the place, enabling designers to leverage design opportunities such as spatial features, objects, and social activities that were taking place. Moreover, in several cases (e.g. C1 and C2), the need for flexibility in use and spatial placement of the visualizations emerged. Without the use of in-situ methods in an ecologically valid context, it is difficult to understand how the target audience will appropriate the situated visualizations, or how the visualizations may need flexibility to account for unexpected circumstances

Situated visualization is grounded in a long history of computing visions and ideas. Kruger's [55] responsive spaces (1977), Weiser's [98] ubiquitous computing (1991) and Fitzmaurize's [31] situated information spaces (1993) were early influential visions. Projects like Prante's Hello.Wall [70], Tollmar's virtually living together lamps [92], the MIT Tangible Media Group's ambient displays [106], Columbia University's work on the Touring Machine [30], and Situated Documentaries [43] are situated visualizations done under different themes and concepts. Similarly, the recent grand challenges in immersive analytics [29] and arguments within the visualization community to move "visualization beyond the desktop" [75] and focus on opening up the domain for casual information visualization [69], public physical data installations [35] and personal visualization [45], suggest an important focus in situated visualization moving forward.

\section{CONCLUSION}

The surveyed corpus, selected cases and historical examples within the research communities highlight "motor themes" [see 54] for discussing and identifying research areas that span the broader literature and research gaps in narrow topical surveys. Expanding on the understanding of situatedness is an important motor theme here, but our work identified additional themes that motivate and inform future work. We emphasise exploring technologies for situated visualization that account for the presented perspectives, paying attention to the aesthetic and material fit when developing situated visualizations and multiple design considerations, ranging from identifying and developing methods that fit the perspectives to examples of design processes that can better inform design and practitioners. These present fruitful avenues for research and new applications to drive situated visualization forward.

Situated visualization sits at a sweet spot between technical, conceptual, and empirical research. With many technologies and theoretical traditions to build on, it has the potential to develop as a promising area at the intersection of multiple research communities with a common interest in bringing visualization into people's everyday environments.

\section{ACKNOWLEDGMENTS}

We thank Susanne Bødker for providing feedback, Ida Larsen-Ledet for title suggestions, and David Ledo for helping with Table 1. 


\section{REFERENCES}

[1] W. Aigner, S. Miksch, H. Schumann, and C. Tominski. Visualization of Time-Oriented Data. Springer, 2011.

[2] F. Alallah, Y. Sakamoto, and P. Irani. Exploring the Need and Design for Situated Video Analytics. In Proc. SUI '20. ACM, 2020.

[3] B. Bach, R. Dachselt, S. Carpendale, T. Dwyer, C. Collins, and B. Lee. Immersive analytics: Exploring future interaction and visualization technologies for data analytics. In Proc. ISS'16, pages 529-533. ACM, 2016.

[4] J. Bird and Y. Rogers. The Pulse of Tidy Street: Measuring and Publicly Displaying Domestic Electricity Consumption. In Pervasive '10 Workshop: Energy Awareness \& Conservation through Pervasive Applications, 2010. Video: https://www . youtube.com/watch? $\mathrm{v}=\mathrm{v}-\mathrm{L} 5$ helmgDo.

[5] S. Bødker. Through the interface-a human activity approach to user interface design. DAIMI Report Series, 16(224), 1991.

[6] S. Bødker, H. Korsgaard, and J. Saad-Sulonen. A Farmer, a Place and at Least 20 Members: The Development of Artifact Ecologies in Volunteer-Based Communities. In Proc. CSCW'16, page 1142-1156. ACM, 2016

[7] N. Bressa. Data In Situ: A Design Approach for Situated Visualization. PhD thesis, Aarhus University, May 2021.

[8] N. Bressa, K. Wannamaker, H. Korsgaard, W. Willett, and J. Vermeulen. Sketching and Ideation Activities for Situated Visualization Design. In Proc. DIS '19, page 173-185. ACM, 2019.

[9] W. Büschel, A. Mitschick, and R. Dachselt. Here and Now: RealityBased Information Retrieval: Perspective Paper. In Proc. CHIIR'18, page 171-180. ACM, 2018.

[10] G. Caggianese, V. Colonnese, and L. Gallo. Situated Visualization in Augmented Reality: Exploring Information Seeking Strategies. In Proc. SITIS '19, pages 390-395, 2019.

[11] J. M. Carroll and M. B. Rosson. Wild at Home: The Neighborhood as a Living Laboratory for HCI. ACM Trans. Comput.-Hum. Interact., 20(3), July 2013.

[12] Z. Chen, W. Tong, Q. Wang, B. Bach, and H. Qu. Augmenting Static Visualizations with PapARVis Designer. In Proc. CHI '20, page 1-12. ACM, 2020.

[13] Ciolfi, Luigina and Bannon, Liam J. Space, place and the design of technologically-enhanced physical environments. In Spaces, spatiality and technology, pages 217-232. Springer, 2005.

[14] S. Claes, J. Coenen, and A. V. Moere. Conveying a Civic Issue through Data via Spatially Distributed Public Visualization and Polling Displays. In Proc. NordiCHI '18, page 597-608. ACM, 2018

[15] S. Claes and A. V. Moere. The Role of Tangible Interaction in Exploring Information on Public Visualization Displays. In Proc. PerDis 15, page 201-207. ACM, 2015.

[16] S. Claes and A. Vande Moere. Street infographics: Raising awareness of local issues through a situated urban visualization. In Proc. PerDis '13, pages 133-138. ACM, 2013.

[17] J. Coenen, P. Biedermann, S. Claes, and A. V. Moere. The Stakeholder Perspective on Using Public Polling Displays for Civic Engagement. In Proc. $C \& T$ '21, C\&T '21, page 61-74, New York, NY, USA, 2021. Association for Computing Machinery.

[18] J. Coenen, M. Houben, and A. Vande Moere. Citizen Dialogue Kit: Public Polling and Data Visualization Displays for Bottom-Up Citizen Participation. In Proc. DIS '19 Companion, page 9-12. ACM, 2019.

[19] J. Coenen, E. Nofal, and A. Vande Moere. How the Arrangement of Content and Location Impact the Use of Multiple Distributed Public Displays. In Proc. DIS '19, page 1415-1426. ACM, 2019.

[20] S. Coulson, M. Woods, M. Scott, D. Hemment, and M. Balestrini. Stop the Noise! Enhancing Meaningfulness in Participatory Sensing with Community Level Indicators. In Proc. DIS '18, page 1183-1192. ACM, 2018

[21] L. A. de Macêdo Morais, N. Andrade, D. M. Costa de Sousa, and L. Ponciano. Defamiliarization, Representation Granularity, and User Experience: A Qualitative Study with Two Situated Visualizations. In Proc. PacificVis '19, pages 92-101, 2019.

[22] P. Dourish. What We Talk about When We Talk about Context. Per sonal Ubiquitous Comput., 8(1):19-30, Feb. 2004.

[23] P. Dourish. Re-space-ing place: "place" and "space" ten years on. In Proc. CSCW '06, CSCW' 06, page 299-308, New York, NY, USA, 2006. ACM.

[24] A. Ducros, C. N. Klokmose, and A. Tabard. Situated sketching and enactment for pervasive displays. In Proc. ISS '19, page 217-228. ACM, 2019

[25] R. Elliott and L. Timulak. Descriptive and Interpretive Approaches to Qualitative Research. A Handbook of Research Methods for Clinical and Health Psychology, 1(7):147-159, 2005.

[26] N. Elmqvist and P. Irani. Ubiquitous analytics: Interacting with big data anywhere, anytime. Computer, 46(04):86-89, apr 2013.

[27] N. A. ElSayed, B. H. Thomas, K. Marriott, J. Piantadosi, and R. T. Smith. Situated Analytics: Demonstrating Immersive Analytical
Tools with Augmented Reality. Journal of Visual Languages \& Computing, 36:13-23, 2016

[28] U. Engelke, H. Hutson, H. Nguyen, and P. de Souza. MelissAR: Towards Augmented Visual Analytics of Honey Bee Behaviour. In Proc. CHI EA'16, page 2057-2063. ACM, 2016.

[29] B. Ens, B. Bach, M. Cordeil, U. Engelke, M. Serrano, W. Willett, A. Prouzeau, C. Anthes, W. Büschel, C. Dunne, T. Dwyer, J. Grubert, J. H. Haga, N. Kirshenbaum, D. Kobayashi, T. Lin, M. Olaosebikan, F. Pointecker, D. Saffo, N. Saquib, D. Schmalstieg, D. A. Szafir, M. Whitlock, and Y. Yang. Grand Challenges in Immersive Analytics. In Proc. CHI'21, New York, NY, USA, 2021. Association for Computing Machinery.

[30] S. Feiner, B. MacIntyre, T. Höllerer, and A. Webster. A Touring Machine: Prototyping 3D Mobile Augmented Reality Systems for Exploring the Urban Environment. Pers. Tech., 1(4):208-217, 1997.

[31] G. W. Fitzmaurice. Situated Information Spaces and Spatially Aware Palmtop Computers. Commun. ACM, 36(7):39-49, 1993.

[32] G. Fitzpatrick. The Locales Framework: Understanding and Designing for Wicked Problems, volume 1. Springer, 2003

[33] A. Fonnet and Y. Prié. Survey of immersive analytics. IEEE TVCG, 27(3):2101-2122, 2019.

[34] M. Francia, M. Golfarelli, and S. Rizzi. A-BI+: A Framework for Augmented Business Intelligence. Information Systems, 92:101520, 2020.

[35] C. Golsteijn, S. Gallacher, L. Koeman, L. Wall, S. Andberg, Y. Rogers, and L. Capra. VoxBox: A tangible machine that gathers opinions from the public at events. In Proc. TEI'15, pages 201-208, 2015 .

[36] P. Gourlet and T. Dassé. Cairn: A Tangible Apparatus for Situated Data Collection, Visualization and Analysis. In Proc. DIS '17, page 247-258. ACM, 2017.

[37] S. Greenberg. Context as a Dynamic Construct. Hum. Comput. Interact., 16(2-4):257-268, 2001.

[38] S. Greenberg, N. Marquardt, T. Ballendat, R. Diaz-Marino, and M. Wang. Proxemic Interactions: The New Ubicomp? interactions, 18(1):42-50, 2011

[39] R. Guarese, P. Andreasson, E. Nilsson, and A. Maciel. Augmented situated visualization methods towards electromagnetic compatibility testing. Computers \& Graphics, 94:1-10, 2021.

[40] R. Guarese, J. a. Becker, H. Fensterseifer, M. Walter, C. Freitas, L. Nedel, and A. Maciel. Augmented situated visualization for spatial and context-aware decision-making. In Proc. AVI '20, New York, NY, USA, 2020. ACM.

[41] G. Gurvitch. The Spectrum of Social Time. Springer, 2012

[42] S. Harrison and P. Dourish. Re-place-ing space: The roles of place and space in collaborative systems. In Proc. CSCW'96, page 67-76. ACM, 1996

[43] T. Hollerer, S. Feiner, and J. Pavlik. Situated documentaries: Embedding multimedia presentations in the real world. In Proc. ISWC '99, pages 79-86, 1999 .

[44] S. Houben, C. Golsteijn, S. Gallacher, R. Johnson, S. Bakker, N. Marquardt, L. Capra, and Y. Rogers. Physikit: Data engagement through physical ambient visualizations in the home. In Proc. $\mathrm{CHI}$ '16, page 1608-1619. ACM, 2016.

[45] D. Huang, M. Tory, B. Aseniero, L. Bartram, S. Bateman, S. Carpendale, A. Tang, and R. Woodbury. Personal Visualization and Personal Visual Analytics. IEEE TVCG, 21(3):420-433, 2015.

[46] C. Hull and W. Willett. Building with Data: Architectural Models as Inspiration for Data Physicalization. In Proc. $\mathrm{CHI}$ '17, page 1217-1264. ACM, 2017

[47] Y. Jansen, P. Dragicevic, P. Isenberg, J. Alexander, A. Karnik, J. Kildal, S. Subramanian, and K. Hornbæk. Opportunities and Challenges for Data Physicalization. In Proc. CHI'15, page 3227-3236. ACM 2015.

[48] R. José, N. Otero, and J. Cardoso. Dimensions of Situatedness for Digital Public Displays. Advances in HCI, 2014, 2014.

[49] D. Kalkofen, C. Sandor, S. White, and D. Schmalstieg. Visualization Techniques for Augmented Reality. In Handbook of Augmented Reality, pages 65-98. Springer, 2011.

[50] V. Kaptelinin. Activity Theory. In The Encyclopedia of HumanComputer Interaction, 2nd Ed., chapter 16. Interaction-design.org, 2021 .

[51] F. Kawsar, J. Vermeulen, K. Smith, K. Luyten, and G. Kortuem. Exploring the Design Space for Situated Glyphs to Support Dynamic Work Environments. In Proc. Pervasive '11, pages 70-78. Springer, 2011

[52] L. Koeman, V. Kalnikaité, and Y. Rogers. "Everyone Is Talking about It!": A Distributed Approach to Urban Voting Technology and Visualisations. In Proc. CHI'15, page 3127-3136. ACM, 2015.

[53] H. Korsgaard. Toward Place-Centric Computing: Making Place With Technology Together. PhD thesis, Aarhus University, Feb. 2017.

[54] V. Kostakos. The big hole in hci research. Interactions, 22(2):48-51, Feb. 2015. 
[55] M. W. Krueger. Responsive environments. In Proc. the National Computer Conference, pages 423-433, 1977.

[56] S. Kush, M. Nicolas, A.-L. Fayard, and V. Bill. CAIRN: A Tangible Research Tool To Materially Visualize Practices in a Makerspace. IJAMM, 2020. https://ijamm.pubpub.org/pub/d2559ieu.

[57] A. Lau and A. Vande Moere. Towards a model of information aesthetics in information visualization. In Proc. IV '07, pages 87-92, 2007

[58] B. Lee, M. Lee, J. Mogk, R. Goldstein, J. Bibliowicz, and A. Tessier. Designing a Multi-Agent Occupant Simulation System to Support Facility Planning and Analysis for COVID-19. In Proc. DIS '21, DIS '21, page 15-30, New York, NY, USA, 2021. Association for Computing Machinery.

[59] N. Li, E. Sharlin, and M. C. Sousa. Duopography: Using back-ofdevice multi-touch input to manipulate spatial data on mobile tangible interactive topography. In Proc. SIGGRAPH Asia '17. ACM, 2017.

[60] T. Lin, R. Singh, Y. Yang, C. Nobre, J. Beyer, M. A. Smith, and H. Pfister. Towards an Understanding of Situated AR Visualization for Basketball Free-Throw Training. In Proc. CHI '21, New York, NY, USA, 2021. Association for Computing Machinery.

[61] M.-J. Lobo and S. Christophe. Opportunities and Challenges for Augmented Reality Situated Geographical Visualization. ISPRS Annals, 4:163-170, 2020.

[62] B. Marques, B. S. Santos, T. Araújo, N. C. Martins, J. B. Alves, and P. Dias. Situated Visualization in The Decision Process Through Augmented Reality. In Proc. IV'19, pages 13-18. IEEE, 2019.

[63] L. Merino, B. Sotomayor-Gómez, X. Yu, R. Salgado, A. Bergel, M. Sedlmair, and D. Weiskopf. Toward Agile Situated Visualization: An Exploratory User Study. In CHI EA '20, page 1-7. ACM, 2020.

[64] A. V. Moere and D. Hill. Designing for the Situated and Public Visualization of Urban Data. J. Urban Technol, 19(2):25-46, 2012.

[65] L. Morais, Y. Jansen, N. Andrade, and P. Dragicevic. Showing Data about People: A Design Space of Anthropographics. IEEE TVCG, pages $1-1,2020$.

[66] D. A. Norman. Technology first, needs last. Interactions, 17(2):38-42, 2010.

[67] T. Pederson. From Conceptual Links to Causal Relations-PhysicalVirtual Artefacts in Mixed-Reality Space. PhD thesis, Umeå University, 2003.

[68] L. J. Perovich, S. Wylie, and R. Bongiovanni. Chemicals in the Creek: Designing a Situated Data Physicalization of Open Government Data with the Community. IEEE TVCG, 27(2):913-923, 2021.

[69] Z. Pousman, J. Stasko, and M. Mateas. Casual Information Visualization: Depictions of Data in Everyday Life. IEEE TVCG, 13(6):1145-1152, Nov. 2007.

[70] T. Prante, C. Röcker, N. Streitz, R. Stenzel, C. Magerkurth, D. Van Alphen, and D. Plewe. Hello.wall-beyond ambient displays. In Adj. Proc. UbiComp '03, pages 277-278, 2003.

[71] A. Prouzeau, Y. Wang, B. Ens, W. Willett, and T. Dwyer. Corsican Twin: Authoring In Situ Augmented Reality Visualisations in Virtual Reality. In Proc. AVI '20. ACM, 2020.

[72] Q. Quach and B. Jenny. Immersive Visualization with Bar Graphics. Cartogr. Geogr. Inf. Sci, 47(6):471-480, 2020.

[73] J. Redström, T. Skog, and L. Hallnäs. Informative Art: Using Amplified Artworks as Information Displays. In Proc. DARE 'OO, page 103-114. ACM, 2000.

[74] W. Reitberger, C. Obermair, B. Ploderer, A. Meschtscherjakov, and M. Tscheligi. Enhancing the Shopping Experience with Ambient Displays: A Field Study in a Retail Store. In Ambient Intelligence, pages 314-331. Springer, 2007.

[75] J. C. Roberts, P. D. Ritsos, S. K. Badam, D. Brodbeck, J. Kennedy, and N. Elmqvist. Visualization beyond the desktop-the next big thing. IEEE CG\&A, 34(6):26-34, Nov 2014.

[76] J. Rodgers and L. Bartram. Exploring Ambient and Artistic Visualization for Residential Energy Use Feedback. IEEE TVCG, 17(12):2489-2497, Dec 2011

[77] Y. Rogers and P. Marshall. Research in the wild. Synth. Lect. Hum. Centered Inf., 10(3):i-97, 2017.

[78] R. Rynasiewicz. Newton's Views on Space, Time, and Motion. In The Stanford Encyclopedia of Philosophy. Stanford University, summer edition, 2014.

[79] M. Satkowski and R. Dachselt. Investigating the Impact of RealWorld Environments on the Perception of 2D Visualizations in Augmented Reality. In Proc. CHI '21, New York, NY, USA, 2021. Association for Computing Machinery.

[80] G. Schall, S. Zollmann, and G. Reitmayr. Smart Vidente: Advances in Mobile Augmented Reality for Interactive Visualization of Underground Infrastructure. Pers. Ubiquitous Comput., 17(7):1533-1549, Oct. 2013.

[81] B. Schilit, N. Adams, and R. Want. Context-Aware Computing Applications. In WMCSA '94, pages 85-90. IEEE, 1994.

[82] D. Schmalstieg and T. Höllerer. Augmented Reality: Principles and
Practice. Addison-Wesley Professional, 2016.

[83] A. Schmidt, M. Beigl, and H.-W. Gellersen. There is More to Context than Location. Computers \& Graphics, 23(6):893-901, 1999.

[84] J. Schoffelen, S. Claes, L. Huybrechts, S. Martens, A. Chua, and A. V. Moere. Visualising Things. Perspectives on How to Make Things Public through Visualisation. CoDesign, 11(3-4):179-192, 2015.

[85] R. Sicat, J. Li, J. Choi, M. Cordeil, W. Jeong, B. Bach, and H. Pfister DXR: A Toolkit for Building Immersive Data Visualizations. IEEE TVCG, 25(1):715-725, 2019.

[86] S. Singhal, W. Odom, L. Bartram, and C. Neustaedter. Time-Turner: Data Engagement Through Everyday Objects in the Home. In Proc. DIS '17 Companion, page 72-78. ACM, 2017.

[87] J. Stasko, T. Miller, Z. Pousman, C. Plaue, and O. Ullah. Personalized Peripheral Information Awareness Through Information Art. In Proc. UbiComp '04, pages 18-35. Springer, 2004.

[88] L. A. Suchman. Plans and Situated Actions: The Problem of HumanMachine Communication. Cambridge University Press, 1987.

[89] M. Tatzgern. Situated visualization in augmented reality. $\mathrm{PhD}$ thesis, Graz University of Technology, 2015.

[90] B. H. Thomas, G. F. Welch, P. Dragicevic, N. Elmqvist, P. Irani, Y. Jansen, D. Schmalstieg, A. Tabard, N. A. ElSayed, R. T. Smith, et al. Situated Analytics. Immersive Analytics, 11190:185-220, 2018.

[91] B. Thompson, L. Levy, A. Lambeth, D. Byrd, J. Alcaidinho, I. Radu, and M. Gandy. Participatory design of STEM education AR experiences for heterogeneous student groups: exploring dimensions of tangibility, simulation, and interaction. In 2016 IEEE International Symposium on Mixed and Augmented Reality (ISMAR-Adjunct), pages 53-58. IEEE, 2016.

[92] K. Tollmar, S. Junestrand, and O. Torgny. Virtually Living Together. In Proc. DIS '00, page 83-91. ACM, 2000.

[93] Y.-F. Tuan. Space and place: humanistic perspective. In Philosophy in geography, pages 387-427. Springer, 1979.

[94] N. Valkanova, S. Jorda, and A. Vande Moere. Public Visualization Displays of Citizen Data: Design, Impact and Implications. Int. J. Hum. Comput. Stud., 81:4 - 16, 2015.

[95] J. Vermeulen, F. Kawsar, A. L. Simeone, G. Kortuem, K. Luyten, and K. Coninx. Informing the Design of Situated Glyphs for a Care Facility. In Proc. VL/HCC '12, pages 89-96. IEEE, 2012.

[96] J. A. Walsh and B. H. Thomas. Visualising Environmental Corrosion in Outdoor Augmented Reality. In Proc. AUIC '11, pages 39-46. Australian Computer Society, 2011.

[97] K. A. Wannamaker, S. Z. G. Kollannur, M. Dörk, and W. Willett. I/O Bits: User-Driven, Situated, and Dedicated Self-Tracking. In Proc. DIS '21, DIS '21, page 523-537, New York, NY, USA, 2021. Association for Computing Machinery.

[98] M. Weiser. The Computer for the 21st Century. Scientific American, 265(3):94-105, 1991.

[99] M. Weiß, K. Angerbauer, A. Voit, M. Schwarzl, M. Sedlmair, and S. Mayer. Revisited: Comparison of Empirical Methods to Evaluate Visualizations Supporting Crafting and Assembly Purposes. IEEE TVCG, 27(2):1204-1213, 2021

[100] S. White and S. Feiner. SiteLens: Situated Visualization Techniques for Urban Site Visits. In Proc. CHI '09, page 1117-1120. ACM, 2009.

[101] S. White, S. Feiner, and J. Kopylec. Virtual Vouchers: Prototyping a Mobile Augmented Reality User Interface for Botanical Species Identification. In Proc. 3DUI '06, pages 119-126. IEEE, 2006.

[102] S. White, D. Feng, and S. Feiner. Interaction and Presentation Techniques for Shake Menus in Tangible Augmented Reality. In Proc. ISMAR '09, pages 39-48, 2009.

[103] M. Whitlock, D. A. Szafir, and K. Gruchalla. HydrogenAR: Interactive Data-Driven Presentation of Dispenser Reliability. In Proc. ISMAR '20, pages 704-712, 2020.

[104] M. Whitlock, K. Wu, and D. A. Szafir. Designing for Mobile and Immersive Visual Analytics in the Field. IEEE TVCG, 26(1):503$513,2020$.

[105] W. Willett, Y. Jansen, and P. Dragicevic. Embedded Data Representations. IEEE TVCG, 23(1):461-470, Jan. 2017.

[106] C. Wisneski, H. Ishii, A. Dahley, M. G. Gorbet, S. Brave, B. Ullmer, and P. Yarin. Ambient Displays: Turning Architectural Space into an Interface between People and Digital Information. In Proc. CoBuild '98, page 22-32. Springer-Verlag, 1998

[107] A. Zimmermann, A. Lorenz, and R. Oppermann. An Operational Definition of Context. In International and Interdisciplinary Conference on Modeling and Using Context, pages 558-571. Springer, 2007.

[108] S. Zollmann, C. Poglitsch, and J. Ventura. VISGIS: Dynamic Situated Visualization for Geographic Information Systems. In Proc. IVCNZ '16, pages $1-6,2016$. 